\title{
Striational Muscle Antibody Positivity Heralding a Diagnosis of Autoimmune Encephalitis Associated with Epiglottic Squamous Cell Carcinoma: A Case Report
}

\author{
By Arun Swaminathan \\ University of Nebraska Medical Center
}

Abstract- A 59 yo woman presented to our emergency room with confusion, spells of loss of awareness over four weekswith malaise, weakness, gait difficulties, and $30 \mathrm{lb}$ weight loss over one year. Brain imaging was unremarkable. EEGs revealed diffuse slowing, triphasic GPDs, and left temporal epileptiform discharges. Spinal fluid showed an elevated protein count. She received a working diagnosis of autoimmune encephalitis and received steroids and antiepileptic medications. She experienced an improvement in symptoms and went home. Follow up revealed elevated striational muscle antibodies and enhancement of spinal nerve roots suggesting an autoimmune neurological syndrome. She received intravenous immune globulin (IVIg) therapy and had periods of improvement followed by worsening a few months later with each dose of IVIg. She presented to the hospital a year with worsening dysphagia and an endoscopy showing an epiglottic mass. Pathology confirmed squamous cell carcinoma, and she received chemotherapy and radiation. We wish to share this rare case of autoimmune encephalitis presenting with only striational antibodies as a heralding sign of a tumor. We postulate that, in the correct context, the presence of striational antibodies alone, despite low titers, may support a diagnosis of autoimmune encephalitis from underlying malignancy.

Keywords: epilepsy, autoimmune encephalitis, striational antibodies.

GJMR-F Classification: NLMC Code: WP 460

Strictly as per the compliance and regulations of:

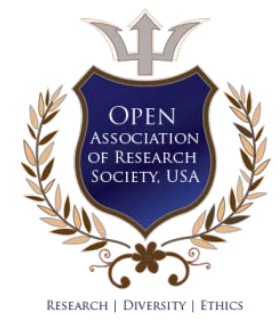

(c) 2020. Arun Swaminathan. This is a research/review paper, distributed under the terms of the Creative Commons AttributionNoncommercial 3.0 Unported License http://creativecommons.org/licenses/by-nc/3.0/), permitting all non-commercial use, distribution, and reproduction in any medium, provided the original work is properly cited. 


\title{
Striational Muscle Antibody Positivity Heralding a Diagnosis of Autoimmune Encephalitis Associated with Epiglottic Squamous Cell Carcinoma: A Case Report
}

\author{
Arun Swaminathan
}

Abstract- A 59 yo woman presented to our emergency room with confusion, spells of loss of awareness over four weekswith malaise, weakness, gait difficulties, and $30 \mathrm{lb}$ weight loss over one year. Brain imaging was unremarkable. EEGs revealed diffuse slowing, triphasic GPDs, and left temporal epileptiform discharges. Spinal fluid showed an elevated protein count. She received a working diagnosis of autoimmune encephalitis and received steroids and antiepileptic medications. She experienced an improvement in symptoms and went home. Follow up revealed elevated striational muscle antibodies and enhancement of spinal nerve roots suggesting an autoimmune neurological syndrome. She received intravenous immune globulin (IVIg) therapy and had periods of improvement followed by worsening a few months later with each dose of IVIg. She presented to the hospital a year with worsening dysphagia and an endoscopy showing an epiglottic mass. Pathology confirmed squamous cell carcinoma, and she received chemotherapy and radiation. We wish to share this rare case of autoimmune encephalitis presenting with only striational antibodies as a heralding sign of a tumor. We postulate that, in the correct context, the presence of striational antibodies alone, despite low titers, may support a diagnosis of autoimmune encephalitis from underlying malignancy.

Keywords: epilepsy, autoimmune encephalitis, striational antibodies.

Abbreviations

EEG - electroencephalogram

GPDs - Generalized Periodic Discharges

GRDA - Generalized Rhythmic Delta Activity

IVIg - Intravenous Immune Globulin

CSF - Cerebrospinal Fluid

ENT - Ear Nose Throat doctors -otorhinolaryngologists

\section{Case Presentation}

1 59 yo right-handed woman was brought into our emergency room by her family with multiple complaints. She had intermittent spells of confusion lasting for a few minutes with associated word-finding difficulty and staring as well, which occurred 2-3 times a week, on average. She also

Author: MD - Assistant Professor of Neurology, Epilepsy Division, University of Nebraska Medical Center (UNMC) - Department of Neurology Omaha, NE, USA. e-mail: arun.swaminathan@unmc.edu complained of generalized weakness and fatigue, which had been slowly worsening over the last year. She also reported difficulty with walking and endorsed back pain in addition to diffuse muscle weakness. She had a remote history of breast cancer 18 years ago, for which she had undergone resection and chemotherapy.

MRI imaging of her brain was unremarkable. Video EEG testing revealed diffuse polymorphic slowing with intermittent generalized rhythmic delta activity (GRDA) in addition to triphasic generalized periodic discharges (GPDs) and occasional left temporal epileptiform discharges (Figures 1, 2, 3). These findings were felt to be suggestive of a diffuse cerebral dysfunction and new-onset epileptogenic potential as well. Spinal fluid analysis revealed elevated protein level of $61 \mathrm{mg} / \mathrm{dL}$ (normal range - 15-45 mg/dL). CSF cytology was negative for malignancy, and there were no unique oligoclonal bands in the CSF either. CAT scans of her body did not reveal a malignancy. ESR and CRP were elevated at 84 and 18, respectively. We diagnosed her with possible autoimmune encephalitis, given her clinical context with the EEG and CSF findings. She was treated with intravenous steroid therapy and antiepileptic medications (levetiracetam and lacosamide) and returned to normal mental baseline in a few days. She was discharged home and scheduled for outpatient follow up.

Further workup revealed the presence of striational muscle antibodies at a titer of 1:480 in the blood. CSF autoimmune panel was negative. Other auto-antibodies were negative as well. MRI imaging of her spine showed subtle enhancement of her spinal nerve roots, suggestive of a possible inflammatory process. EMG/NCV testing with repetitive nerve stimulation revealed a chronic ulnar neuropathy but no other findings. Imaging and autoimmune antibody evaluation did not capture any abnormalities.

As her symptoms returned a few weeks later, we decided to initiate therapy with intravenous immune globulin (IVIg) for a probable autoimmune neurological syndrome - autoimmune encephalitis without any features of peripheral neuromuscular hyperexcitability. She received three courses of IVIg over the next 1 year 
and had significant improvement in her symptoms, both mental (impaired awareness, confusion) and physical (weakness, gait difficulty). She also did not have any noticeable seizures and remained on levetiracetam and lacosamide therapy during this time.

One year after her first presentation, she presented to her PCP with complaints of worsening dysphagia. She was referred to the ENT doctors for evaluation and underwent an endoscopy, which discovered an epiglottic mass of about $1 \mathrm{~cm}$ diameter. We performed a biopsy and histopathology confirmed diagnosis of squamous cell carcinoma of her epiglottis (Figures 4, 5). Her 40-pack year smoking habit was contributory to her malignancy.

She saw oncologists and received chemotherapy and radiation. She remains stable, continues to receive antiepileptic medications and immune therapy for her symptoms, and follows up with us in the epilepsy clinic.

\section{Discussion}

Autoimmune encephalitis is an uncommon presentation of malignancy. Presenting symptoms are often diverse, including but not limited to, memory loss, personality changes, seizures, cognitive impairment, and overall loss of function ${ }^{1}$. The presentation is often subacute and subtle, with symptoms occurring insidiously over weeks to months before family members or friends note frank presentation. Steroid therapy is often therapeutic, produces dramatic improvement, and frequently serves as a diagnostic and therapeutic intervention in such patients. Our patient's symptoms, while consistent with many of the previously described features of autoimmune encephalitis, was still nonspecific.

Isolated striational antibody positivity is not a classic finding in patients with autoimmune encephalitis. The antibody is typically associated with peripheral hyperexcitability syndromes, like Isaac's or Morvan's syndromes or myasthenia gravis. It is seen in patients with autoimmune encephalitis in coexistence with neuromuscular syndromes, especially with elevated titers of other autoantibodies ${ }^{2,3,4}$. Titers of striational autoantibodies are also felt to play a role, with lower titers usually seen to represent evidence of autoimmunity, rather than the presence of an underlying malignancy, especially with the absence of other autoantibodies ${ }^{5}$. A striational antibody titer of at least $1: 7680$ is reportedly suggestive of an underlying malignancy, especially in conjunction with antibodies like VGKC complex, GAD 65, or others. Prior malignancy is causative of a low titer of striational antibodies, rather than a current active malignancy ${ }^{5}$. Our patient had a relatively low titer of striational antibodies at 1:480, which, coupled with her previous diagnosis of breast cancer, did not initially support the probability of a newer malignancy. Absence of a amalignancy on the chest and abdominal imaging further suggested a lower possibility of a tumor, since lung and thymus tumors are most commonly associated with paraneoplastic syndromes ${ }^{3,5,6}$. EMG/NCV testing ruled out peripheral hyperexcitability as well, making autoimmune encephalitis less likely, ${ }^{3,}$.

We approached the diagnostic dilemma and treatment plans for patients with a presumptive diagnosis of possible autoimmune encephalitis. Our patient had subacute onset of symptoms inclusive of encephalopathy and confusion, new-onset seizures, and a reasonable exclusion of other causes, meeting all three criteria for a diagnosis of possible autoimmune encephalitis (Table 1) ${ }^{7}$. We decided to pursue empirical therapy with intravenous steroids due to the potential benefits and relatively low probability of risk in this clinical context. Our patient responded to steroid therapy, which seemed to strengthen our presumptive diagnosis of autoimmune encephalitis. We do concede that other conditions, like lymphomas, would also respond to steroid therapy. Still, we were reasonably confident that such diagnoses could be excluded based on the negative results seen on our extensive testing. Immune globulin therapy represents the standard of care, and she had responses to multiple courses of IVIg followed by a slow progression of symptoms over weeks-months after treatment, supporting our working diagnosis of autoimmune encephalitis. Her EEG tests did show diffuse slowing with triphasic waves and left temporal epileptiform discharges - findings that would favor diffuse cerebral dysfunction and new-onset seizures. While these findings were not specific for autoimmune encephalitis, they did add supportive evidence to the diagnosis of possible autoimmune encephalitis.

\section{Table 1: Diagnostic criteria for possible autoimmune encephalitis ${ }^{7}-$}

\begin{tabular}{|l|l|}
\hline Diagnosis can be made when all three of the following criteria have been met: \\
\hline 1) Subacute onset (rapid progression of less than 3 months) of working memory deficits (short-term memory loss), \\
altered mental status, or psychiatric symptoms \\
\hline 2) At least one of the following: \\
- New focal CNS findings \\
- Seizures not explained by a previously known seizure disorder \\
- CSF pleocytosis (white blood cell count of more than five cells per $\mathrm{mm}^{3}$ ) \\
\hline 3) Reasonable exclusion of alternative causes \\
\hline
\end{tabular}


Epiglottic tumors are not commonly associated with autoimmune neurological syndromes, and our workup did not reveal such a tumor during our initial round of testing, including whole-body PET scans. Lung cancers and thymomas are most commonly associated with autoimmune encephalitis and striational antibodyrelated paraneoplastic neurological syndromes ${ }^{6}$, and we were able to exclude these relatively common conditions with our imaging tests, especially given her history of smoking.

In summary, we can state that our patient represents a rare case of autoimmune encephalitis presenting with subacute symptoms, heralded by a diagnosis of positive striational autoantibodies, in association with epiglottic cancer. All these findings constitute a rare constellation of results and symptoms and make this patient's case worthy of publication and scientific study.

\section{ili. Conclusions}

Autoimmune encephalitis must be considered in any patients presenting with subacute cerebral dysfunction and new-onset seizures. Extensive cerebral and whole-body imaging and additional testing are essential to confirm the diagnosis and exclude other conditions as well. We recommend empirical steroid or immune therapy at the earliest due to the potential for improvement with minimal potential risk. Consultation by an expert neurologist, epileptologist, oncologist, or rheumatologist is also advised at the earliest, especially if the patient is unstable or rapidly declining. Serial imaging and surveillance are often required before the diagnosis is confirmed and may take months to years to achieve. Empirical immune therapy must be considered with correct clinical and serological guidance and should probably be performed under expert consultation only.

\section{ACKNOWLEDGMENTS}

I thank Challa Lowry, EEG tech, UNMC, for her assistance in compiling the EEG screenshots. I am grateful to Ernesto Martinez Duarte MD MPH, Dept. of Pathology, UNMC, for his guidance in collecting and reproducing the excellent quality histopathological pictures.

\section{Funding}

There was no funding involved in this case report.

\section{Permissions and guidelines}

The patient and her family consented to the publication of this case report. All scientific and ethical guidelines were followed during the compiling and publication of this report and this publicationis consistent with those accepted guidelines.

\section{References Références Referencias}

1. Schott JM, Harkness K, Barnes J, et al. Amnesia, cerebral atrophy, and autoimmunity. Lancet 2003; 361:1266.

2. Pittock SJ, et al. Paraneoplastic antibodies coexist and predict cancer, not neurological syndrome. Ann Neurol 2004; 56 (5), 715-9.

3. Rickman $\mathrm{OB}$, et al. Fulminant Autoimmune Cortical Encephalitis Associated With Thymoma Treated With Plasma Exchange. Mayo ClinProc 2000; 75 (12), 1321-6.

4. Thieben MJ, et al. Potentially reversible autoimmune limbic encephalitis with neuronal potassium channel antibody. Neurology 2004; 62 (7), 1177-82.

5. McKeon A, Lennon VA, et al. Striational Antibodies in a Paraneoplastic Context. Muscle Nerve 2013; 47: 585-587.

6. Gozzard P, Woodhall M, Chapman C, et al. Paraneoplastic neurologic disorders in small cell lung carcinoma: a prospective study. Neurology 2015; 85:235-9.

7. Graus F, et al. A clinical approach to diagnosis of autoimmune encephalitis. Lancet Neurol. 2016 Apr; 15(4):391-404. 
PQ

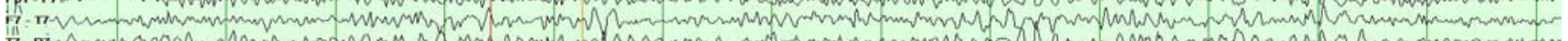

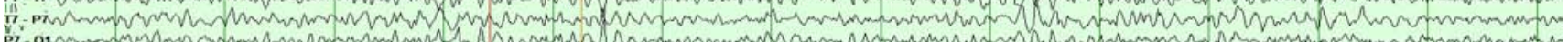
F Fpe is is 年,

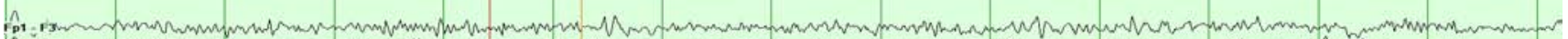

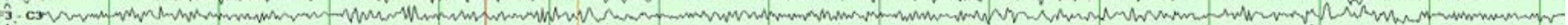

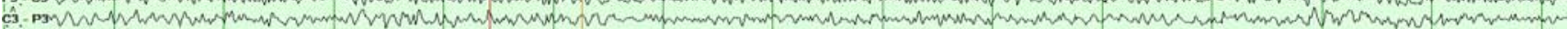

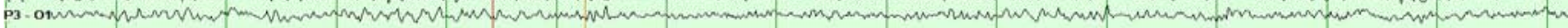

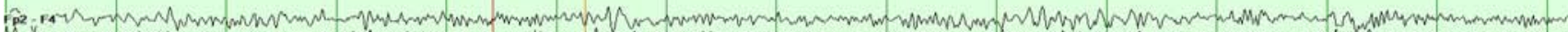

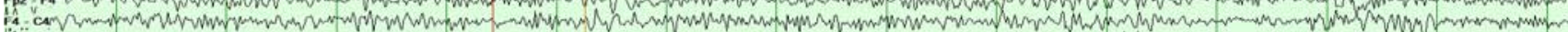
c.

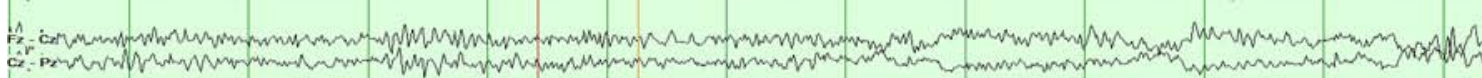

EKGL-EKGR RY

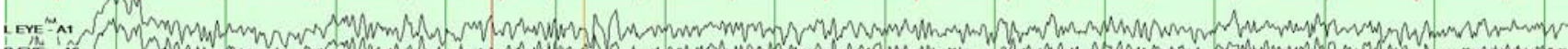
NE

Figure 1: EEG with double banana montage showing a left temporal epileptiform discharge

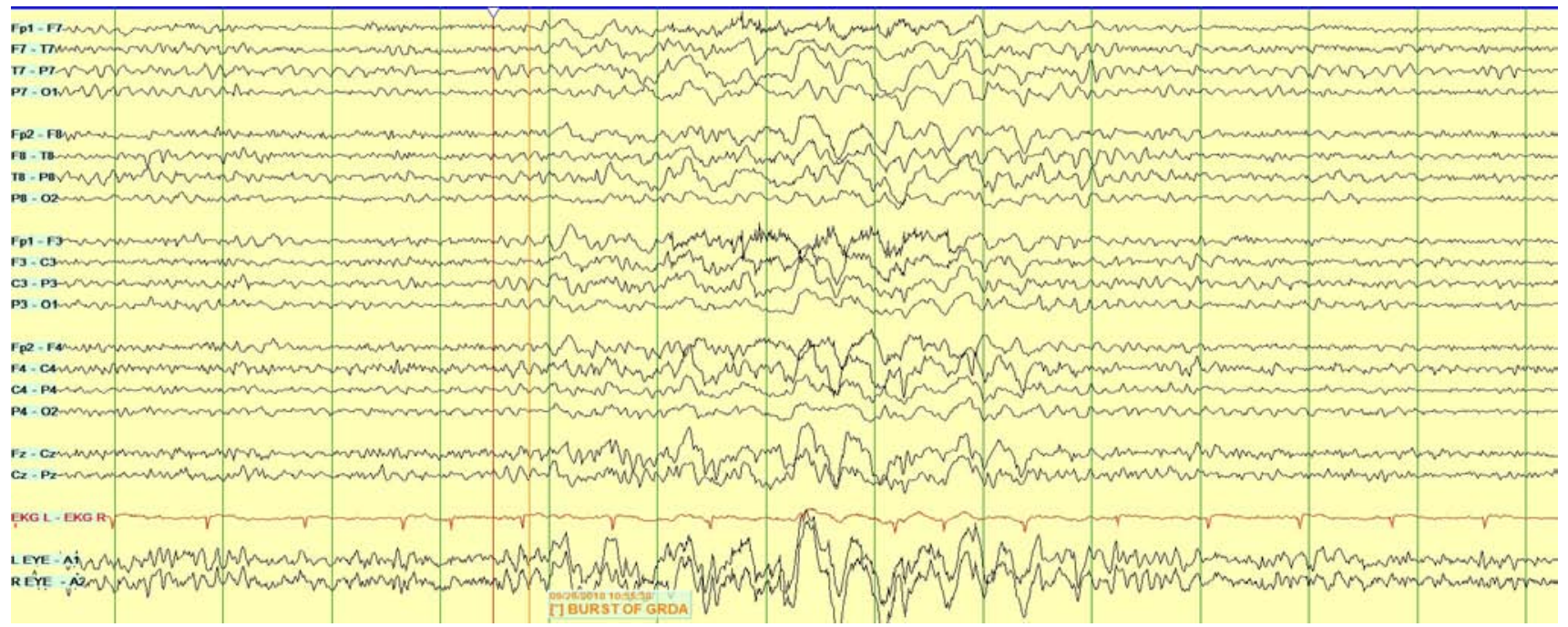

Figure 2: EEG with double banana montage showing generalized rhythmic delta activity - GRDA - with diffuse polymorphic slowing

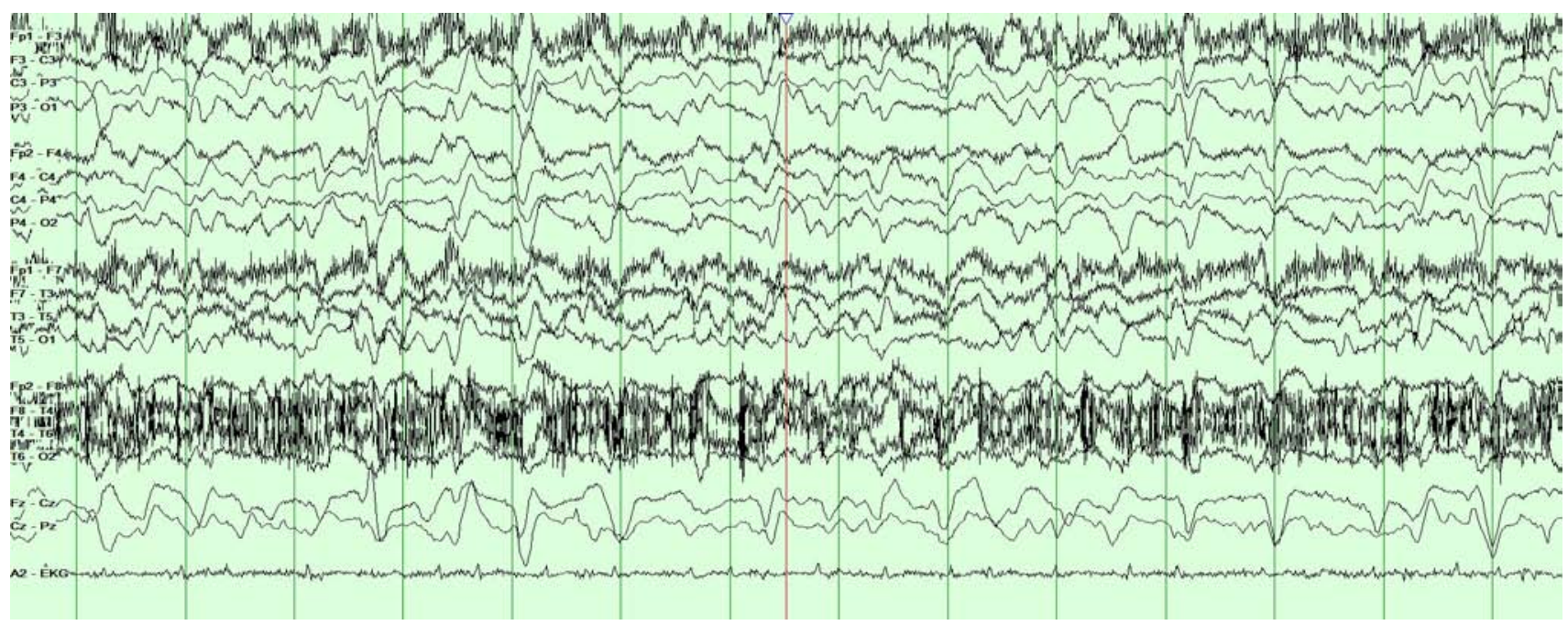

Figure 3: EEG with double banana montage showing generalized periodic discharges - GPDs - with triphasic morphology at $1-2 \mathrm{~Hz}$ 

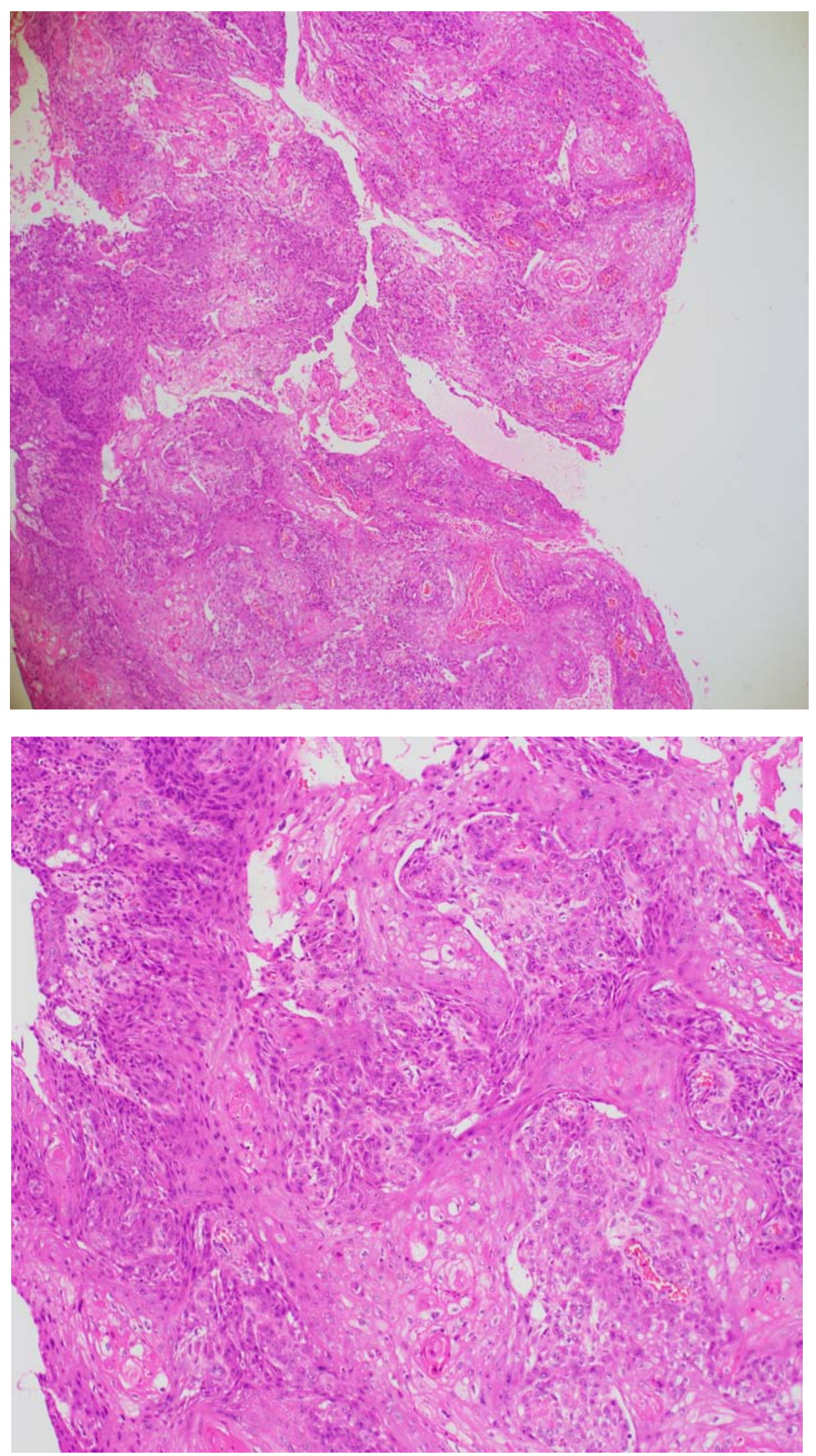

Figures 4 \& 5: Histopathology of epiglotticlesion - Invasive squamous cell carcinoma of the epiglottis with infiltrative growth, keratinization, moderate nuclear pleomorphism and scattered mitotic figures. Fig 4 (H\&E 10X) \& Fig 5 